\title{
EDITORIAL
}

\section{The use of interferon- $\gamma$-based blood tests for the detection of latent tuberculosis infection}

\author{
P.D.O. Davies* and F. Drobniewski ${ }^{\#}$
}

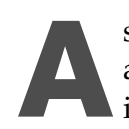
s recently as 3 yrs ago, a colleague complained that after 100 yrs the only way to detect tuberculosis (TB) infection was still to measure "bumps on arms". Now, at last, we have a genuine improved alternative: the ex vivo cellular interferon (IFN)- $\gamma$-based blood tests. The crucial questions we need to ask are how good they are at detecting infection with the TB bacterium and whether they are costeffective? Unfortunately, determining the answers to these questions is not easy.

The new ex vivo cellular IFN- $\gamma$ assays (CIGAs) have been developed based on the release of IFN- $\gamma$ from a patient's Tcells when exposed to mycobacterial antigens. These novel assays rely on the principle that the genes encoding the secretory proteins early secretory antigenic target (ESAT)6/ culture filtrate protein (CFP) 10 are absent in the bacille Calmette-Guérin (BCG) vaccine strain and most environmental mycobacteria [1]. Two commercial cellular immunological assays have been developed in which IFN- $\gamma$ output from the patient's whole blood or peripheral blood mononuclear cells are measured following treatment with ESAT6/CFP10 antigens [1].

Different assay formats have been developed. One method relies on the relatively straightforward ELISA detection after a fixed volume of whole blood is incubated in a tube with ESAT6/CFP10 antigens (Quantiferon Gold; Cellestis Limited, Carnegie, Australia). The other method currently available commercially is the T-Spot.TB assay (Oxford Immunotec, Oxford, UK) based on the enzyme-linked immunospot (ELISPOT) principle. The methods appear to be both sensitive and specific. These assays have the potential to change the face of the diagnosis of latent TB infection (LTBI) and active TB at an early stage in the disease process. Their use is also likely to transform the screening of new migrants and other population groups. These tests require only a single blood sample without the repeat visit required for the tuberculin skin test (TST), thus reducing costs within the screening service (or increasing the throughput of existing staff). However, these tests will increase laboratory costs.

\footnotetext{
*Tuberculosis Research and Resources Centre, Liverpool, " Health Protection Agency National Mycobacterium Reference Unit, Center for Infectious Diseases, Institute for Cell and Molecular Sciences, Barts and the London School of Medicine, London, UK.
}

CORRESPONDENCE: P.D.O. Davies, Tuberculosis Research and Resources Centre, Thomas Drive, Liverpool L14 3PE, UK. Fax: 44 1512932254. E-mail: peter.davies@ctc.nhs.uk
The assays are time dependent in that the blood samples need to be transferred to the laboratory within a few hours. The throughput of the Quantiferon Gold (QFN-G) assay is higher than the T-Spot.TB test and the new QFN-G in-tube system (QFN-GT) is much less time sensitive. Conversely, the QFNGT appears to be less sensitive than the T-Spot.TB system.

Active TB disease itself can be very difficult to diagnose. The gold standard of a positive culture is often not forthcoming. Data from the UK show that only $68 \%$ of pulmonary patients treated for TB are culture proven with only $49 \%$ of extrapulmonary cases and an even lower proportion in children [2]. In other words, a third of pulmonary cases and a half of extrapulmonary cases who have been treated for TB in the UK have no proof of diagnosis according to the accepted gold standard of culture positivity. These assays are not as sensitive for diagnosing active TB since IFN responses decline as TB develops, but are likely to be of value in diagnosing TB in children and in immunocompromised individuals where the sensitivity of existing methods is low.

How accurate are these new blood tests in detecting LTBI? There is no absolute gold standard for LTBI equivalent to the positive culture in the case of TB disease. Nevertheless, TSTs have formed the bedrock of our understanding of TB infection in populations and in contact tracing of exposed individuals. In community investigations, workers have had to employ a combination of TST and careful epidemiological assessment of exposure to the index case and the degree of infectivity of that case. Now, the new CIGAs have been added to these traditional tools.

In this edition of the European Respiratory Journal (ERJ), a total of five papers investigating these tests are published.

DiEl et al. [3] have described a potential outbreak around a single index case in a police academy in Germany. A total of 369 contacts were screened of whom 36 could be defined as close and 333 as casual contacts. All were given a TST and the positive cases, defined as having $\geqslant 5 \mathrm{~mm}$ of induration, were offered an ELISPOT test. Only 13 of the 36 close contacts had a positive test on ELISPOT testing, indicating its value in eliminating the false positives that are revealed with a history of previous BCG vaccination. However, this advantage was considerably reduced if the cut-off point for a positive TST was taken to be $\geqslant 10 \mathrm{~mm}$ rather than $\geqslant 5 \mathrm{~mm}$ of induration.

LEE et al. [4] have prospectively compared the two CIGAs with the TST in a small study of 79 individuals with active TB, some in an immunocompromised state and a control group of high 
school students who were unlikely to have LTBI. In the active disease group, the TST was $72 \%$ sensitive compared with $96 \%$ for the T-Spot.TB test and 77\% for the QFN-G test. In the immunocompromised group, the T-Spot.TB test was $100 \%$ sensitive (24 out of 24 ) compared with $75 \%$ for the QFT-G test and $37 \%$ for the TST. Conversely, in the control group, the QFN-G test (92\%) showed better specificity over the TST (82\%) and the T-Spot.TB test (86\%). The difference between the QFN$\mathrm{G}$ test and the TST was statistically significant.

The paper by PIANA et al. [5] compared the TST against the TSpot.TB test alone in a group of 138 immunocompromised haematology patients who had potential exposure to an infectious TB case. They found $44 \%$ were positive, and thus likely to be infected, using the T-Spot.TB test compared with $17 \%$ for the TST. The use of TST was unreliable in this group but the T-Spot.TB test was unaffected by immunosuppression (or less affected since direct exposure of patients to the index case was difficult to determine with accuracy, and three individuals were TST positive but T-Spot.TB negative). None of the screened contacts had developed TB at 1 yr of follow-up.

A tentative conclusion to draw from these papers might be that the greater sensitivity of the T-Spot.TB test makes it the better test to rule in LTBI or active disease in immunocompromised populations, whereas the slightly higher specificity of the QFT$G$ test may make it marginally better at ruling out infection or disease. However, a degree of caution must be exercised here as there was no statistically significant difference found in specificity between the two tests.

Other studies have shown that the CIGAs are likely to have greater sensitivity and specificity than the TST [6, 7]. So which test should we use? At present, there seems to no clear advantage in test performance and so differences in the number of tests performed per operator, the proportion of tests giving an indeterminate result, and maximum time to reach the laboratory for processing are likely to be the most important factors.

The consensus seems to be that the QFN-G test marketed by Cellestis is technically easier to carry out but less sensitive, particularly in the immunosuppressed patient, and with a higher incidence of indeterminate results than the ELISPOT TSpot.TB test marketed by Oxford Immunotech. The greater throughput of the samples for QFT-G (and greater stability regarding specimens of the QFT-GT) may make these tests more suitable for general contact tracing, but, where individuals are immunocompromised, the T-spot.TB test would be more accurate; the QFT-GT contains an additional antigen that may increase the sensitivity of the assay.

It is clear that more studies are needed to compare the two tests available, particularly for the diagnosis of active TB in children, the immunocompromised and specialised groups of patients, such as those prior to organ transplant and those about to receive anti-tumour necrosis factor therapy.

The American guidelines [8] on the investigation of persons with possible LTBI state that the QFN-G CIGA (which is Food and Drug Administration approved) may be used instead of the TST (and it is reasonable to extend these findings to the T-Spot.TB test): "CDC [Centers for Disease Control and
Prevention] recommends that QFT-G can be used in all circumstances in which the TST is currently used, including contact investigations. QFT-G can be used in place of and not in addition to the TST. A positive QFT-G result should prompt the same evaluation and management as a positive TST."

What then of cost-effectiveness? The problem with modelling cost-effectiveness is that the data underpinning the initial assumptions used for the model may be inexact. The first aspect of modelling the CIGAs against the TST is that only one clinic visit needs to be costed compared with the two clinic visits. Secondly, a more accurate diagnosis of LTBI may decrease costs as those who might have been skin-test positive due to BCG and given unnecessary chest radiography or preventive chemotherapy are excluded. Conversely, costs might rise (although in an "appropriate" and cost-effective manner) as those rendered falsely negative on TST due to immunosuppression might be given chest radiography and preventive therapy as the CIGA shows them to have LTBI.

Two papers in this issue of the ERJ have examined the way in which CIGAs can be used cost-effectively $[9,10]$. The fact that one paper models the QFN-G test and the other the T-Spot.TB test makes no difference to the outcomes of the modelling exercise. Both compare the possible models of using the TST alone compared with using the CIGA test alone and using the TST followed by a CIGA test on the TST-positive individuals. The models are sensitive to the number of costly clinic followup visits made, and this is likely to vary more in clinical practice than visits for follow-up of active treatment. The paper by DIEL et al. [9] had a fourth arm, which examined the CIGA test in those who had received previous BCG. Both papers assume similar costs and outcomes. Both seem to firmly advocate carrying out a TST initially, and then a CIGA test on the TST-positive individuals. This is a similar conclusion to the National Institute for Health and Clinical Excellence guidelines from the UK [11].

Although the use of cellular interferon- $\gamma$ assays may turn out to be more expensive than the old tuberculin skin test, it is likely that their greater sensitivity and specificity will provide for better healthcare and will make a major contribution to the control and eventual elimination of tuberculosis.

\section{REFERENCES}

1 Andersen P, Munk ME, Pollock JM, Doherty TM. Specific immune-based diagnosis of tuberculosis. Lancet 2000; 356: 1099-1104.

2 Health Protection Agency. Tuberculosis. www.hpa.org. $\mathrm{uk} /$ infections/topics_az/tb/menu.htm. Date last updated: December 2005. Date last accessed: February 242006.

3 Diel R, Ernst M, Döscher G, et al. Avoiding the effect of BCG vaccination in detecting Mycobacterium tuberculosis infection with a blood test. Eur Respir J 2006; 28: 16-23.

4 Lee JY, Choi HJ, Park I-N, et al. Comparison of two commercial interferon- $\gamma$ assays for diagnosing Mycobacterium tuberculosis infection. Eur Respir J 2006; 28: 24-30.

5 Piana F, Codecasa LR, Cavallerio P, et al. Use of a T-cellbased test for detection of tuberculosis infection among immunocompromised patients. Eur Respir J 2006; 28: 31-34. 
6 Ewer K, Deeks J, Alvarez L, et al. Comparison of T-cellbased assay with tuberculin skin test for diagnosis of Mycobacterium tuberculosis infection in a school tuberculosis outbreak. Lancet 2003; 361: 1168-1173.

7 Mori T, Sakatani M, Yamagishi F, et al. Specific detection of tuberculosis infection: an interferon-gamma-based assay using new antigens. Am J Respir Crit Care Med 2004; 170: 59-64.

8 Morbidity and Mortality Weekly Report. Recommendations from the National Tuberculosis Controllers Association and CDC Guidelines for the Investigation of Contacts of Persons with Infectious Tuberculosis December 16, 2005 /54(RR15);1-37.www.cdc.gov/mmwr/preview/mmwrhtml/ rr5415a1.htm. Date last updated: December 16, 2006; Date last accessed: February 21, 2006.

9 Diel R, Nienhaus A, Lange C, Schaberg T. Cost-optimisation of screening for latent tuberculosis in close contacts. Eur Respir J 2006; 28: 35-44.

10 Wrighton-Smith P, Zellweger J-P. Direct costs of three models for the screening of latent tuberculosis infection. Eur Respir J 2006; 28: 45-50.

11 Tuberculosis: clinical diagnosis and management of tuberculosis, and measures for its prevention and control. A clinical guideline for the NHS in England and Wales. National Collaborating Centre for Chronic conditions the Royal College of Physicians (In Press). 